\title{
Ivermectin and onchocerciasis: is it all solved?
}

\begin{abstract}
The donation of ivermectin by Merck and Co. Inc. has led to one of the most effective private-public partnerships controlling a disease of major public health importance particularly in Africa. The up scaling of ivermectin distribution during the last 15 years has been remarkable with almost 40 million people treated in 2003, many already on their regular annual dose. The tools that have been developed particularly by the APOC programme have been instrumental in this increase. However, ivermectin is a microfilaricide and does not kill the adult worms. Distribution will be needed for at least 25 years and latest estimates indicate that 90 million people need annual treatment if onchocerciasis is to be eliminated as a public health problem. In spite of this incredible progress it is difficult to see how the programme will be sustained, especially after the closure of APOC in 2010. A macrofilaricide destroying adult worms and safe for mass distribution would solve the problem of onchocerciasis.
\end{abstract}

Eye (2005) 19, 1057-1066. doi:10.1038/sj.eye.6701962

Keywords: onchocerciasis; ivermectin; sustainability; conflict areas; coendemicity

\section{Introduction}

Approximately 100 million people are at risk of Onchocerciasis. Most of these people live in Africa. It has been calculated that 40000 new cases of blindness occur each year.

Approximately 300000 are blind and 500000 visually impaired. ${ }^{1}$ In some communities, the prevalence of blindness can be well over $2 \%$, with adults often in their $30 \mathrm{~s}$ going blind. ${ }^{2}$ Expectation of life is also reduced by 8-12 years. Ivermectin, a micro-filaricide that can prevent blindness is available but needs to be given annually. Merck and Co. Inc. have donated
AD Hopkins

Mectizan (ivermectin) free to all who need it for as long as is necessary. Almost 40 million people received ivermectin in 2003, a remarkable achievement. ${ }^{3}$

In order to control the disease in Africa there are 90 million people who need annual treatment for at least 20 years. Many who need annual ivermectin treatment live at the end of the road in poor rural communities or often beyond the end of the road in the bush, particularly in the war torn countries of Central Africa. In hyperendemic foci it is not unusual to find up to $50 \%$ of adults over 40 years with blindness or severe visual impairment.

If ivermectin distribution remains the only strategy then mass distribution mechanisms will need to be functional for this 20-year plus period. Not all countries are at the same level of development. Most countries in Central Africa are in conflict or in post conflict situations and support for mass distribution programmes particularly from governments will be difficult to maintain in view of multiple health priorities.

Ivermectin will not eradicate onchocerciasis in Africa. ${ }^{4}$ What is urgently needed is a macrofilaricide, to kill adult filariae, safe enough for mass distribution that would enable onchocerciasis to be controlled.

The disease

The life cycle of Onchocerca volvulus is shown in Figure 1. The adult females of $O$. volvulus are found in 'nodules' (Figure 2) swellings, which are often subcutaneous and situated over bony prominences and where many female worms are found intertwined as shown in Figure 1. The females produce thousands of microfilariae (mf) per day, which being mobile invade many parts of the body.

If the $\mathrm{mf}$ are not ingested by a Simulium black fly they die and create a mild inflammatory reaction. The accumulated effect of repeated small foci of inflammation is a gradual
CBM Regional Office, Nairobi, Kenya

Correspondence: AD Hopkins, CBM Regional Office, PO Box 58004,

Nairobi, Kenya

Tel: + 254735717655 , +243 9931354;

Fax: + 254203740305 .

E-mail: ahopkins@

cbmi-nbo.org

Received: 12 April 2005 Accepted: 2 May 2005 


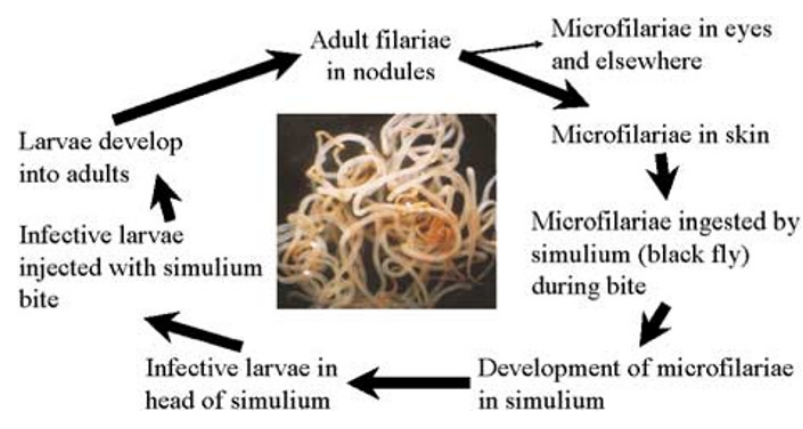

Figure 1 Onchocerciasis: life cycle.

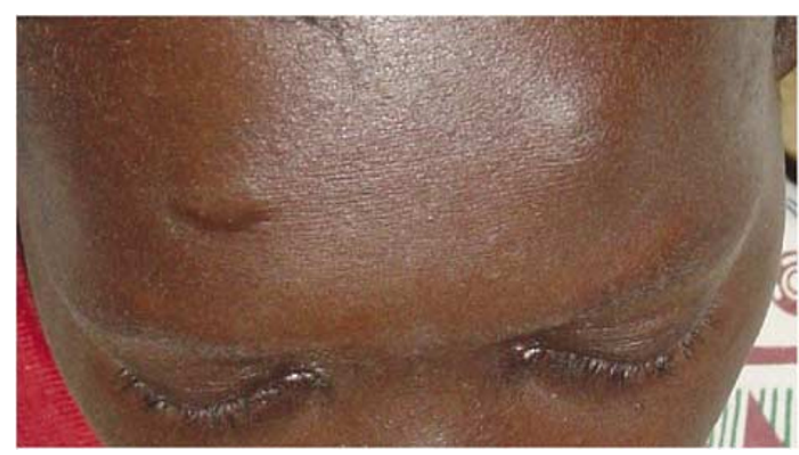

Figure 2 Child with nodule over left eye and scar from removed nodule over right eye.

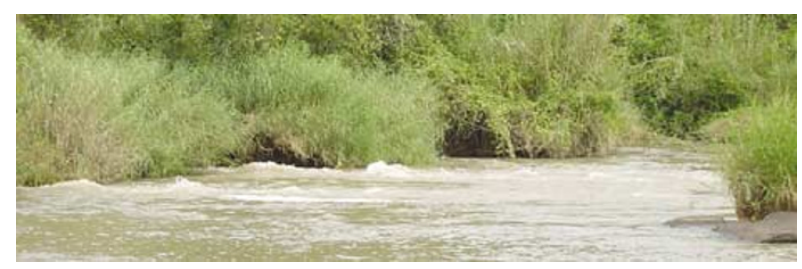

Figure 3 Typical breeding site of Simulium damnosum.

destruction of tissues, clinically important in the eye, skin and brain.

The Simulium breeds in well-oxygenated water (Figure 3) and the female fly requires a blood meal to produce each new batch of eggs. The $\mathrm{mf}$ are transformed in the fly into infective larvae and invade a new host when the female Simulium needs a further blood meal.

The $\mathrm{mf}$ of $O$. volvulus invade the eye and provoke inflammation both in the anterior and posterior segments. Microfilaria in the cornea produce early reversible changes, punctate or snow flake keratitis, but changes can become permanent leading to sclerosing keratitis (Figure 4). In the AC, mf provoke a chronic anterior uveitis and can form a pseudo-hypopion of microfilaria with live $\mathrm{mf}$ shimmering on the surface. Secondary cataract often follows (Figure 4).

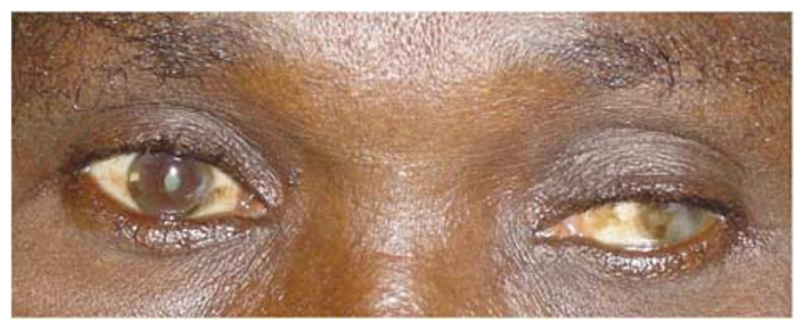

Figure 4 Sclerosing keratitis left eye, chronic uveitis and secondary cataract right eye.

The posterior segment of the eye is equally involved, sometimes with little involvement of the anterior segment allowing a clear view of an increasingly destructive chorio-retinitis with loss of vision, most often beginning at the periphery. The optic nerve can be involved leading to optic atrophy.

Onchocerciasis also provokes inflammation of the skin. Without treatment early reversible inflammatory changes become permanent, giving the typical leopard skin, lizard skin and atrophic changes of the skin. Skin disease can become a cause of social isolation and affects school attendance. Families with severe onchocerciasis spend more money on health care per year but are the poorest in the community.

Although it has been more difficult to prove there are probably significant effects due to $O$. volvulus in the brain. There appears to be a relationship between certain types of epilepsy and severe onchocerciasis but the causal relationship has not yet been clarified, (some epilepsy is improved after taking ivermectin). ${ }^{5}$ Children in badly affected communities also suffer from 'nodding disease' a form of epilepsy creating nodding movements usually stimulated by the presence of food probably due to a high-mf load.

\section{Onchocerciasis control mechanisms}

Control of onchocerciasis is based on an interruption of the life cycle. Ivermectin also controls morbidity due to the disease by reducing the micro-filarial load.

\section{Destroying the adult filaria}

Surgical removal (nodulectomy): This is impossible at a community level due to the millions who will need surgery in a population with a high prevalence of HIV infection. Many patients have nodules deep in the tissues and these are therefore inaccessible to surgery.

Medication to kill the adult filaria (microfilaricide): Suramin kills adult worms but is too toxic for mass distribution. No other macrofilaricide is available at present. 


\section{Eliminating the vector}

Eliminating the Simulium was the cornerstone of the very successful Onchocerciasis Control Programme in West Africa. Transmission of the disease has been halted in West Africa but it was impossible to expand activities. The programme is prohibitively expensive due to costs of (weekly) larviciding. Larviciding does not help patients already heavily infected.

\section{Use of a microfilaricide}

Diethylcarbamazine (DEC) is an effective micro-filaricide but it is very toxic. It provokes visual loss in some patients and is therefore contraindicated for treatment of other filarial diseases in areas where there is also onchocerciasis.

Mectizan ${ }^{\circledR}$ (Ivermectin - Merck) is much less toxic especially to the eye. Ivermectin was donated by Merck in 1987 for as many who needed for as long as was needed. This donation has been one of the most significant drug donations by a pharmaceutical company to combat a widespread disease of major public health importance. ${ }^{6,7}$

Ivermectin is very safe for mass distribution and is effective as a once yearly dose, although small numbers of $\mathrm{mf}$ are produced after 4-6 months after the treatment. It can be given using a simple dose calculation based on height, which can easily be measured in rural communities. Adults live for approximately 14 years but the treatment needs to be taken for 20-25 years or as long as transmission occurs. However, for individuals there is considerable reduction in morbidity due to onchocerciasis after the first treatment, symptoms of itching disappear within a few days and patients describe a feeling of good health that they had not experienced for many years, probably due not only to a reduction in microfilarial load, but due to evacuation of intestinal parasites, head-lice and scabies. There is some improvement in the anterior segment of the eye where irreversible changes have not already occurred. ${ }^{8-10}$ Changes may continue to evolve in the posterior segment. There are reactions to treatment, which are particularly severe in the first year in heavily infected individuals or those with Loa-Loa.

\section{Mass treatment strategies with ivermectin}

Treatment was done initially on an individual basis with strict controls, particularly of hypotension, which had been reported in a few cases. Mass treatment was only carried out where an epidemiological survey based on skin snips had confirmed endemicity of onchocerciasis. With the development of nodule surveys mass treatment became much easier to organise from 1991 and many more mass treatment projects began. ${ }^{11}$

Community diagnosis is based on nodule counts (Figure 5). At the community level, this is called Rapid Epidemiological Assessment (REA). This technique can be used in selected communities to produce a map, Rapid Epidemiological Mapping of Onchocerciasis (REMO) (Figure 6). ${ }^{12}$ Mass treatment is carried out where onchocerciasis is meso- or hyperendemic. Hypoendemic communities are treated on an individual basis by staff at health centres.

Most programmes at the beginning depended on some sort of mobile teams, visiting the communities, carrying out a community diagnosis, and then organising mass treatment if required. Mobile teams are unsustainable over 15-25 years therefore a sustainable approach had to be developed. It has been found that communities once fully informed are capable of organising their own treatment. In fact communities once empowered not only organise but also control and direct their treatment in what is called Community Directed Treatment with Ivermectin (CDTI). The role of the health worker is to communicate the benefits of the programme to the community and then pass on programme management skills to community members.

\section{CDTI community activities ${ }^{13}$}

After suitable Information, Education and Communication (IEC), the communities

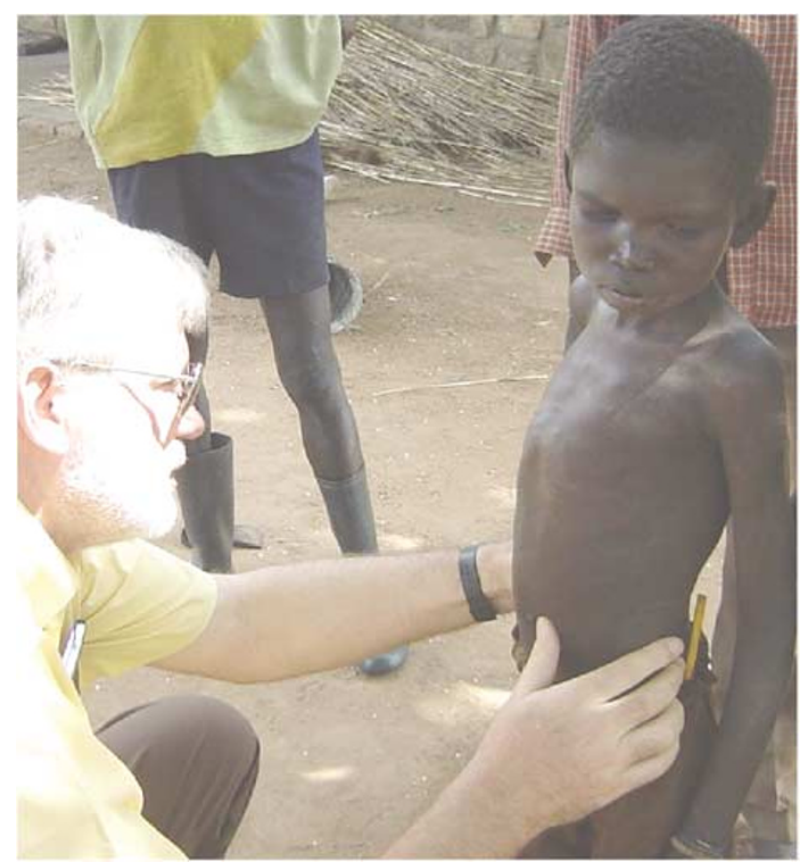

Figure 5 Examining a child for nodules. For REA and REMO only adults over 20 are examined. 


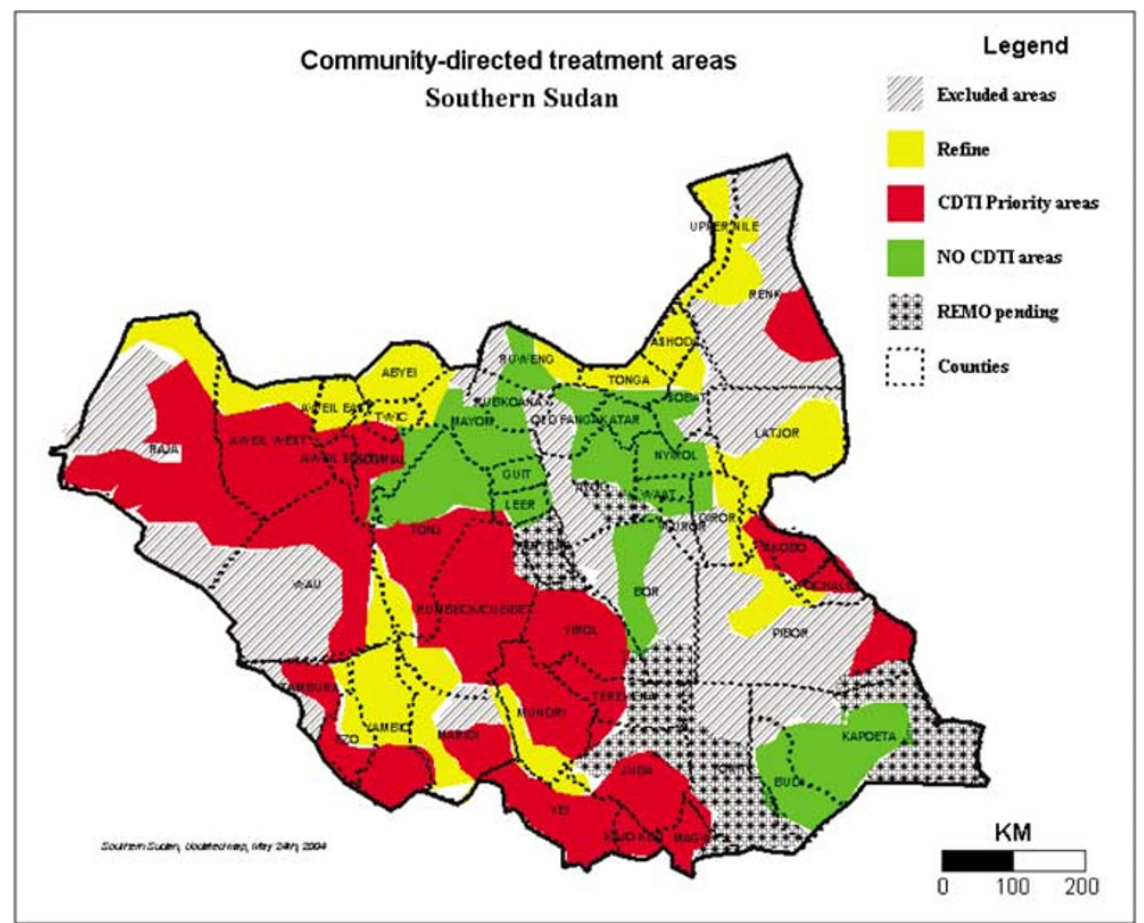

Figure 6 The REMO map for South Sudan, produced by APOC. This is not yet complete but has been produced in spite of the conflict situation and is the basis for the five new projects.

- Choose a distributor for training

- Do a census to calculate ivermectin requirements

- Organise the collection of ivermectin from a Health Centre or other distribution point

- Organise a distribution method, house to house, fixed point in village, etc.

- Help the distributor to calculate the dose and distribute the Ivermectin

- Note the treatment statistics and report to the health authorities

- Participate in community supervision

After 5 years communities should be ready to continue treatment alone with minimal help and supervision from the primary health care (PHC) services. Equally a mechanism is in place for mass distribution of other medicines.

\section{Control programmes for onchocerciasis}

In Central America, where there are only small foci of the disease, the Onchocerciasis Elimination Programme of the Americas (OEPA) is hoping to eradicate the disease using mass distribution of ivermectin twice yearly, although there are also other research regimens being used.

The Onchocerciasis Control Programme (OCP) covered 11 countries in West Africa. OCP used mostly a strategy of Vector control. After the introduction of ivermectin in 1987 this was added in some areas. The programme was concluded in 2002 with activities being devolved to the countries concerned although there are five Special Intervention Zones, including the whole programme in Sierra Leone. All these programmes are continuing with ivermectin distribution where required, mostly supported by Non Governmental Development Organisations (NGDOs).

The African Programme for Onchocerciasis Control, (APOC) is a unique example of partnership created out of the experiences of the NGDOs working in non OPC countries (Figure 7) and building on the expertise and financing of the OCP countries. ${ }^{14}$ Merck donates the ivermectin. A total of, $75 \%$ of financing comes from donor countries into a World Bank Trust Fund. In all, 25\% of the funding comes from the NGDOs. WHO is the executing agency via the APOC management structure based in Ouagadougou. Work in the field is carried out by teams from the respective Ministries of Health, mostly with NGDO support and a major contribution come from the communities themselves. APOC is using mass distribution of ivermectin in mesoendemic and hyperendemic communities as its principal strategy to eliminate the disease as a public health problem. In some isolated foci where there is the chance of eliminating the vector, vector eradication is also being used. APOC together with the Tropical Diseases Research Unit at 


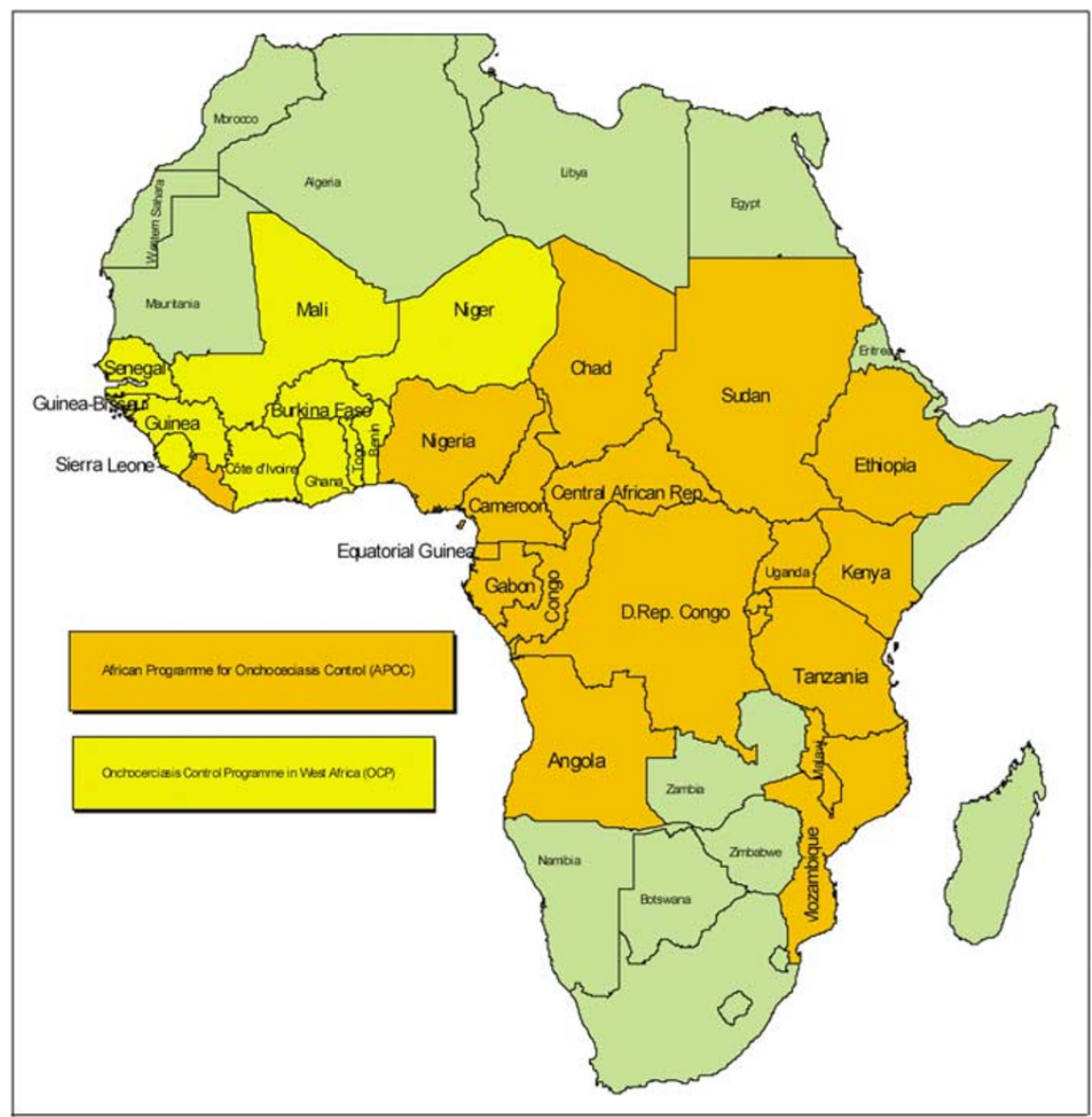

Figure 7 The geographical distribution of African programmes for onchocerciasis control.

WHO (TDR) and other researchers have developed several new strategies, principally mapping of the disease (REMO), the Community Directed approach (Community Directed Treatment with Ivermectin/CDTI) as well as tools for monitoring progress, not only the distribution, but also community involvement. The APOC strategy includes the preparation of sustainability plans in the hope that the Community Directed Strategy put into place will continue beyond the end of APOC support in 2010.

\section{Treatment}

Mass treatments have increased dramatically since 1991 with a modified community approach, and also since the beginnings of APOC field activities in 1996 (Figure 8).

\section{Discussion}

\section{Is the problem of onchocerciasis solved?}

The scaling up of ivermectin distribution over the last 10 years is a major achievement. The population in affected

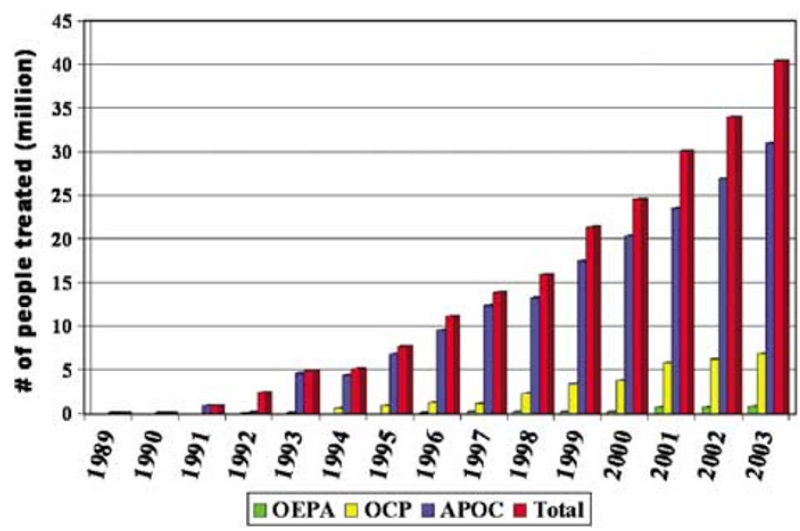

Figure 8 NGDO supported treatment with mectizan ${ }^{\mathbb{R}}$ within the APOC, OCP and OEPA partnerships.

communities has had considerable relief of suffering, both in blindness and skin disease. Fertile land that was deserted due to onchocerciasis is once more under cultivation in West Africa. Community involvement in their own treatment has shown that if communities are given responsibility for their own health they are able to 
carry out the necessary activities. The contribution of the international community to the various programmes has been unprecedented, with many countries donating since the mid-1970s and still contributing. The coordination of activities by the various NGDOs and their role in the various control programmes has been significant. The development of the mapping techniques has clearly defined the populations that need treatment. Tools developed by APOC for monitoring, evaluation and sustainability have led to a much better follow-up of the programmes.

However, the problem of onchocerciasis is not yet solved. There are several issues to be faced if onchocerciasis is to be eliminated as a public health problem.

1. Up scaling: When the APOC programme began at the end of 1995 it was forecast that approximately 45 million people would need treatment. With the improved epidemiological mapping the objective is now twice that number. APOC will finish in 2010. The remaining communities are amongst the most difficult to reach.

2. The length of treatment: When ivermectin was first available it was forecast that treatment would be necessary for 10-15 years. Computer modelling now suggests 20-25 years providing therapeutic coverage is at least $65 \%$ and geographical coverage is $100 \%$, but may be 35 years if coverage is less. Research into an end point for treatment is planned by WHO/TDR but not yet funded.
The degree of this challenge is seen in Figure 9 with treatments extrapolated to 2020 but many treatments will need to continue to 2030. Such a long-term programme and the up scaling required is an ongoing challenge to the onchocerciasis community.

The up scaling and length of treatment required have created major challenges:

1. Funding: It was hoped that by year 6 of an APOC programme the countries using minimal input from Ministries of Health and a major contribution from the communities would be able to continue as shown in Figure 10. Most programmes are receiving limited funding from APOC from year 6 to year 8, as well as from NGDOs. A report to the WHO-NGDO coordination committee in Atlanta (September 2004) showed that most governments are still not contributing to the programme as planned. Is this a lack of interest by the governments concerned or due to a lack of funds and a long list of other health priorities? The projected contribution of government support in Burundi is equal to one third of the total health budget. Is this realistic? Should treatment stop if the governments do not contribute their share?

An example is the programme in CAR (Figure 11). As can be seen, the numbers treated from 1993 increased satisfactorily until the year 2000 in spite of a civil war in 1996/7. However, the contribution of the NGDO (CBM) levelled off after 4 years and remained stable as the NGDO continued to maintain government salaries of the people involved. The prolonged and more violent civil disturbances created further problems from 2002. Having treated for so long is it ethical to stop support in 2005 ?

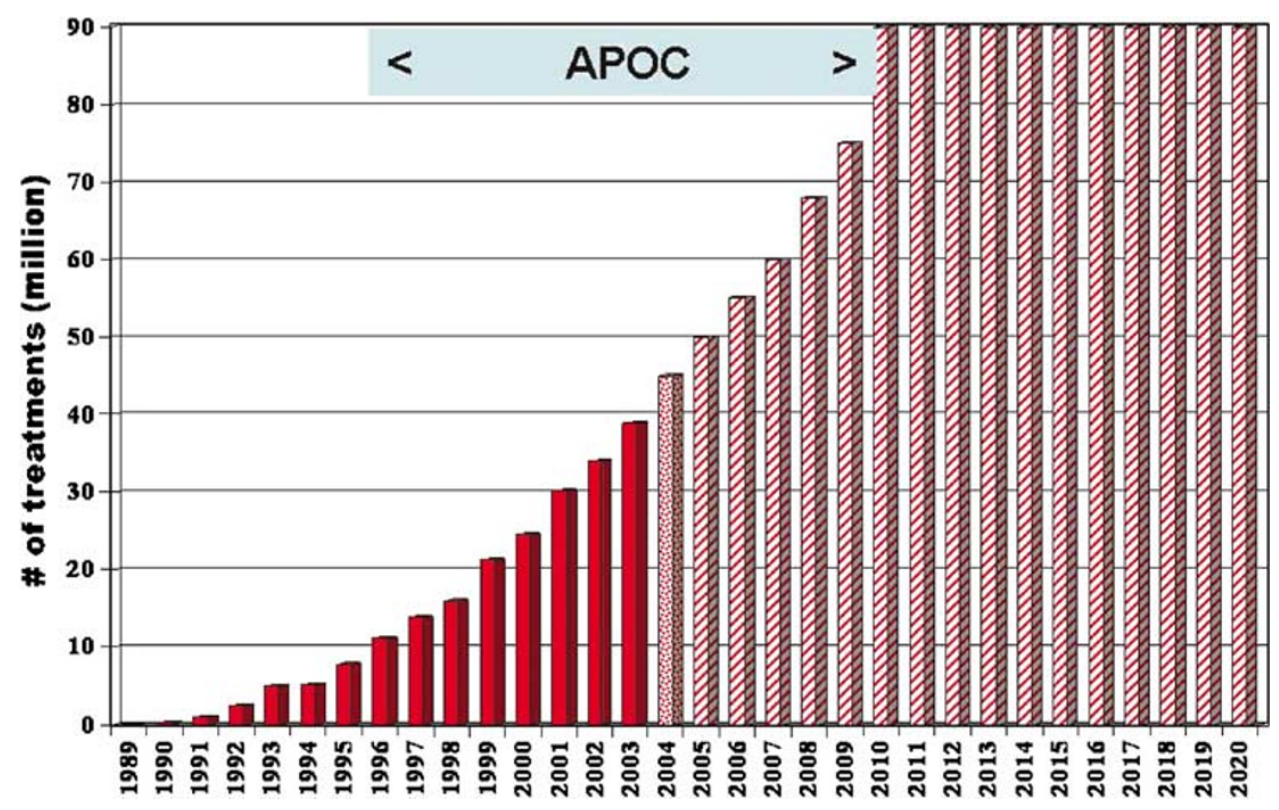

Figure 9 Treatments and projection to 2020. Partner input into control programmes. 


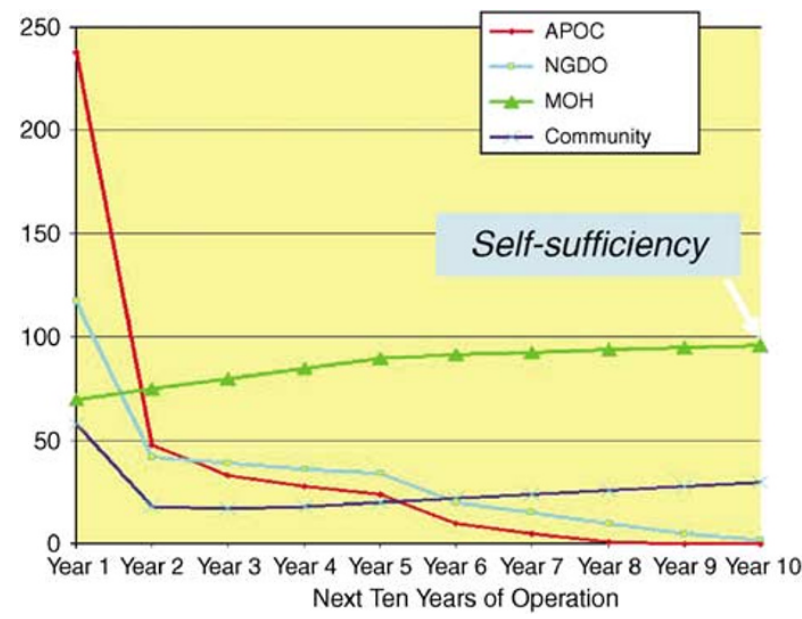

Figure 10 Partner input into control programmes.

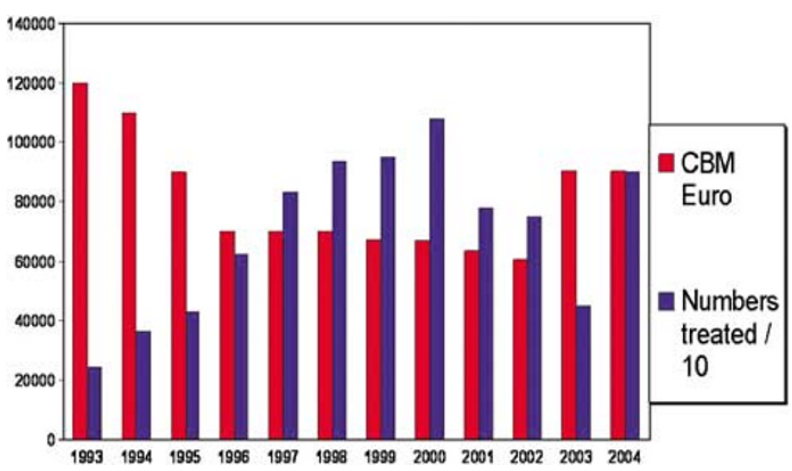

Figure 11 Funding.

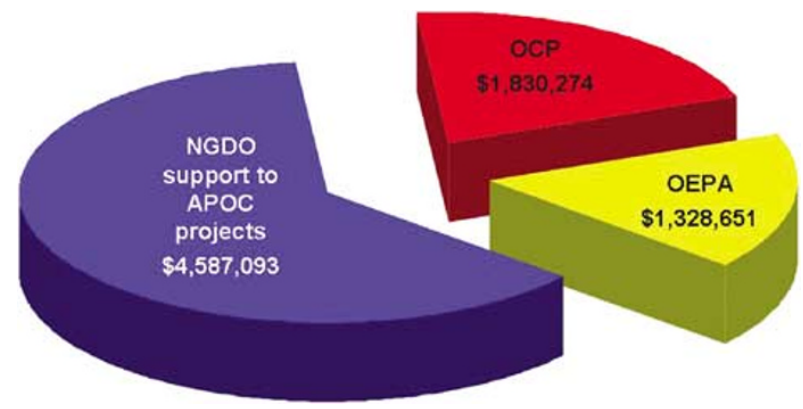

Figure 12 NGDO group financial support-2002.

NGDOs have been funding some mass distribution of ivermectin for 15 years and are still involved in the former OCP countries. With the closure of APOC it is clear that NGDOs will need to be involved in some areas for many more years to come. NGDOs as a group have been funding ivermectin distribution at around 7 million US dollars per year (Figure 12). With competition for resources it is difficult to see how this level of support can be maintained for another 20 years.

\begin{tabular}{|l|r|}
\hline I. Mectizan® & $\$ 309,938,250$ \\
\hline $\begin{array}{l}\text { II. Shipping \& customs } \\
\text { clearance }\end{array}$ & $\$ 210,000$ \\
\hline $\begin{array}{l}\text { III. Program Man. \& } \\
\text { Admin. }\end{array}$ & $\$ 2,065,000$ \\
\hline Grant Total & $\$ 312,213,250$ \\
\hline
\end{tabular}

Total number of tablets shipped: $206,625,500$

Figure 13 Merck oncho support-2003.

Contributions by APOC/World Bank of 12-14 million US dollars per year and NGDOs are completely overshadowed by the support of Merck and Co. Inc. who donated over 200 million tablets of ivermectin in 2003 (Figure 13). Merck have created new production lines to cope with demand and also pay all shipping costs to the main port or airport of the countries concerned. In 2004 they also contributed 1 million dollars to the OEPA programme.

2. Sustainability: In order to maintain ivermectin distribution at a satisfactory level of coverage, it is vital to integrate CDTI into other programmes. CDTI is basically a PHC Activity. Where these services exist CDTI can be fully integrated at little cost as one of the 'minimal health package' activities. However unfortunately in many areas PHC services are poorly developed and it will be difficult to integrate CDTI into these PHC programmes. Eye Care NGDOs have been in the forefront of integration into other activities, for example, mass Vitamin A distribution, and integrated eye care (particularly cataract identification). Other NGDOs have integrated different mass distribution activities into CDTI, for example, control of lymphatic filariasis, schistosomiasis, and malaria (bed nets and treatment), and the sale of condoms, etc.

APOC management have been very active in reviewing sustainability and have developed a series of sustainability evaluation tools. ${ }^{15,16}$ Projects are visited and judged on a series of criteria based on interviews with health staff at all levels and also the community. The government's financial contribution is also assessed as well as the community participation in the programme. These plans have already indicated that some projects are making progress towards sustainability and are proving to be a good evaluation method. Follow up of these plans is now being planned by APOC. So far no project has been left without APOC Trust fund or NGDO support. It is important to test sustainability by stopping support and closely monitoring the result. 
3. Working in conflict areas: Apart from a few foci in Ethiopia the remaining important foci to treat are in DR Congo, Sudan, Angola, Sierra Leone and Liberia. Infrastructure in these countries is mostly neglected or destroyed (Figures 14 and 15), Human Resources are limited and health services are almost non-existent.

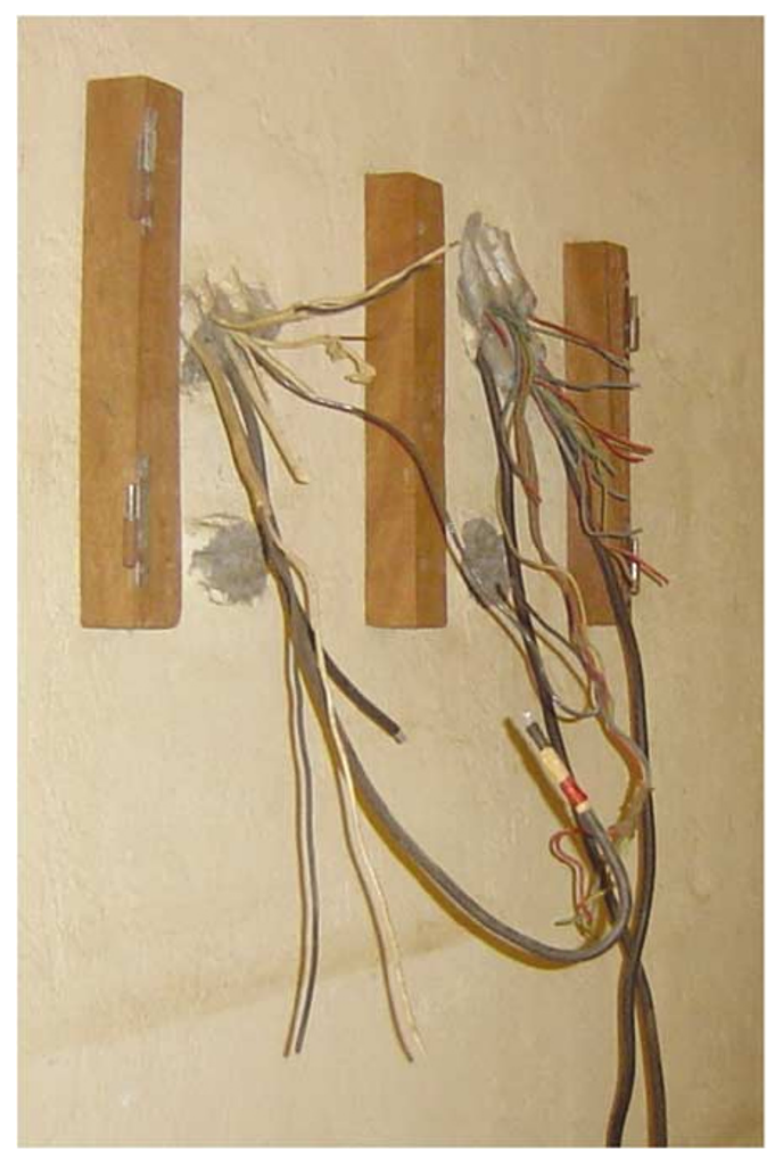

Figure 14 Destruction of infrastructure due to war. All electrical appliances were ripped out from the wall in the Onchocerciasis Control Office in the Central African Republic.

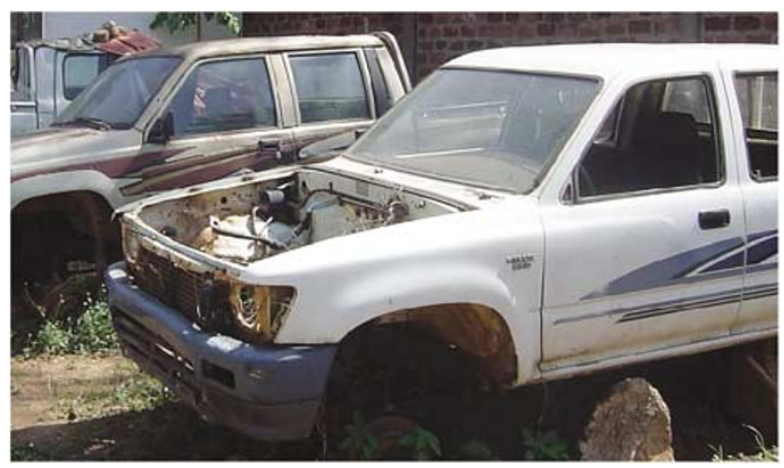

Figure 15 Engine, gearbox and other useful items stolen from an immobilised vehicle during pillaging in Bangui, Central African Republic.
These programmes are therefore more costly and a flexible approach is required. ${ }^{17,18}$ Support cannot be withdrawn after 5 years.

In spite of these problems there is progress in many areas. In South Sudan the current cease fire has enabled much of the mapping (REMO) to be done and 5 new projects are beginning (Figure 6). A workshop was held in Rumbek in South Sudan during the first week of September 2004. The venue was the best accommodation available in the temporary capital city, a tented camp, with a herd of cows outside the enclosure adding to the atmosphere, and UN humanitarian flights taking off regularly over the gazebo used for the workshop. In spite of these difficult circumstances significant progress was made and two out of five projects were ready to start activities in September. It should be mentioned that some ivermectin distribution has been continuing over many years organised by NGDOs in spite of the war.

There is, however, a serious lack of capacity in South Sudan and one of the only two medically trained county medical officers was appointed full time to the onchocerciasis programme.

4. Co-endemicity of onchocerciasis with Loa-Loa Co-infections of onchocerciasis and Loa-Loa can create problems after treatment. ${ }^{19}$ Loa-Loa another filarial parasite is often called the eye worm due to the occasional passage of adult worms across the eye under the conjunctiva. Microfilaria of Loa-Loa are killed by ivermectin. However treatment of patients with high Loa-Loa mf levels risk having severe adverse events. Above 10000 microfilaria per $\mathrm{ml}$ of blood encephalopathy is possible, and above $30000 \mathrm{mf}$ per $\mathrm{ml}$ of blood it is almost certain. Loa-Loa is present in many of the areas remaining to be treated. As with onchocerciasis the risk is most in the first year but is reduced if an annual treatment is given as the $\mathrm{mf}$ load 1 year after Mectizan treatment is much reduced.

A combination of techniques is being used to try to resolve some of the issues. A remote sensing map, developed in Liverpool, from satellite pictures of vegetation indicates probable foci of Loa-Loa (Figure 16). This map is being refined by adding extra data from the field. If the map indicates the possibility of Loa-Loa WHO/TDR have developed a further mapping technique called RAPLOA, which requires village surveys in the area and can give a guide to the possibility of having high microfilarial loads. Only areas of hyperendemic and mesoendemic onchocerciasis, after refinement of the REMO, are treated. These are areas where it is judged that the risk of complications of onchocerciasis justify the treatment with ivermectin in spite of the risks of Loa-Loa encephalopathy.

In areas where there is the risk of encephalopathy a system of transfer to specially equipped centres for the 


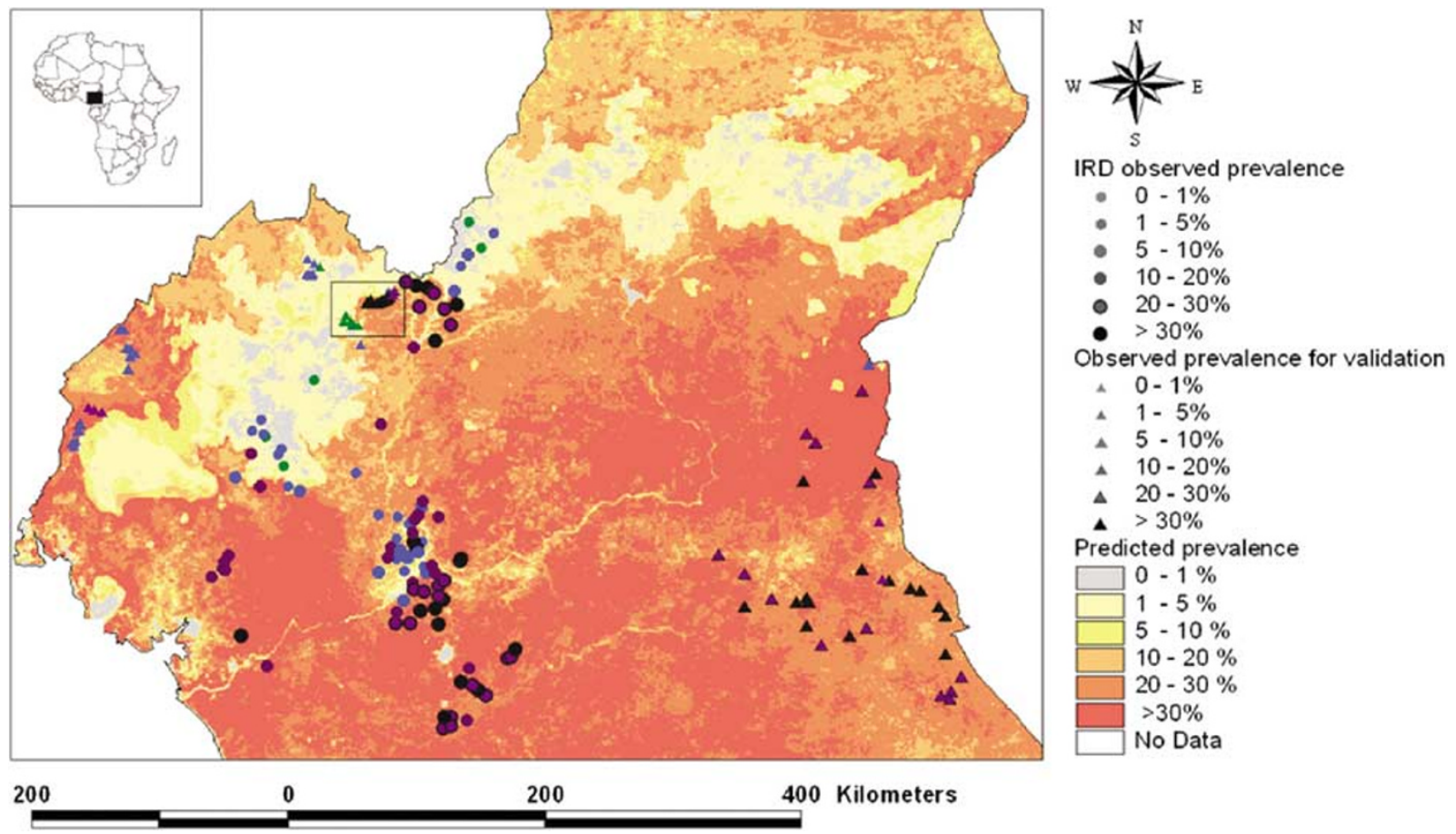

Figure 16 Satellite imagery indicating possible foci of Loa-Loa.

care of unconscious patients is put in place.

Encephalopathy is probably due to micro-emboli of dying Loa-Loa causing cerebral ischemia. The care of patients is supportive, maintaining hydration, preventing decubitus ulcers and treating infections including malaria if they occur. Patients, properly managed, recover consciousness after several days although rehabilitation takes some time. In the extreme west of Congo there were 14 deaths during December 2003. Some of the patients who recovered have neurological sequellae. Unfortunately due to traditional beliefs and transport difficulties patients are not always transferred quickly.

Encephalopathy is often preceded by sub-conjunctival haemorrhages. This sign can be taught to village health workers and be used to encourage early transfer of the patients. Retinal changes also occur with the appearance of Roth's spots.

5. Research for a macro-filaricide: This has been a major activity of both the OCP and the APOC programmes and research is being undertaken by WHO/ TDR. In view of the long period of treatment required with ivermectin to interrupt transmission of the disease, medication to kill the adult worm is essential if onchocerciasis is to be fully controlled.

Moxidectin, an avermectin has shown most promise however it is very similar to ivermectin and may well have a similar action. It is now undergoing trials.
Woolbachia is an endo-parasite living in symbiosis with O. volvulus. If Woolbachiae are destroyed the fertility of the adult female is affected. Doxycycline is useful but would be complicated for mass treatment. Other products are being examined including Azithromycin.

Ivermectin does not appear to destroy adult worms. ${ }^{20}$ Annual treatment with ivermectin has led, however, to a reduction in nodules. This needs further investigation but may indicate that repeated annual ivermectin has some effect on adult worms. ${ }^{10,21,22}$

\section{Conclusion}

Is onchocerciasis solved? The answer is not yet. Since the donation of ivermectin by Merck, major advances have been made in the control of the disease. Many useful tools have been developed which will not only help with the distribution of ivermectin but can be used in the control of other diseases of public health importance. ${ }^{23}$ These need to be sustained over a long period. If a macrofilaricide is developed it can be distributed using the tools that have been developed and the problem of onchocerciasis could then be solved.

\section{Acknowledgements}

The author is employed by Christoffel Blindenmission $\mathrm{eV}$, one of the NGDOs involved in Onchocerciasis 
Control. The map Figures 6 and 7 were provided by APOC with kind permission of the Director Dr A Sékétéli. Figure 16 was reproduced with permission from Thomson MC, Obsomer V, Kamgno J, Gardon J, Wanji S, Takougang I et al. Mapping the distribution of Loa Loa in Cameroon in support of the African Programme for Onchocercisis Control. Filaria J 2004; 3(7).

\section{References}

1 Thylefors B, Negrel A-D, Pararajasegaram R, Dadzie KY. Global data on blindness. Bull World Health Organi 1995; 73(1): 115-121.

2 Schwartz EC, Huss R, Hopkins AD, Dadjim B, Madjitoloum $P$, Henault $C$ et al. Blindness and visual impairement in a region endemic for onchocerciasis in the Central African Republic. Br J Ophthalmol 1997; 81: 443-447.

3 Report of the Twenty First Meeting of the NonGovernmental Development Organisations coordination meeting for Onchocerciasis Control. WHO: Geneva.

4 Final report of the Conference on the Eradicability of Onchocerciasis. Carter Center, Atlanta 2002.

5 Druet-Cabanac M, Preux P-M, Bouteille B, Bernet-Bernady P, Dunanad J, Hopkins A et al. Onchocerciasis and epilepsy: a matched case-control study in the Central African Republic. Am J Epidemiol 1999; 149(6): 565-570.

6 Dull HB, Meredith SE. The Mectizan donation programme - a 10 year report. Ann Trop Med Parsitol 1998; 92(Suppl 1): S73-S77.

7 Peters DH, Philips T. Mectizan donation program: evaluation of a public-private partnership. Trop Med Int Health 2004; 9(Suppl 4): A4-A15.

8 Tielsch JM Beeche A. Impact of ivermectin on illness and disability associated with onchocerciasis. Trop Med Int Health 2004; 9(Suppl 4): A45-A56.

9 Abiose A. Onchocercal eye disease and the impact of Mectizan treatment. Ann Trop Med Parsitol 1998; 92(Suppl 1): S11-S22.

10 Kennedy $\mathrm{MH}$, Bertocchi I, Hopkins AD, Meredith SE. The effect of 5 years of annual treatment with ivermectin (Mectizan) on the prevalence and morbidity of onchocerciasis in the village of Gami in the Central African Republic. Ann Trop Med Parsitol 2002; 96: 297-307.
11 WHO. Strategies for Ivermectin Distribution Through Primary Health Care Systems WHO/PBL/91.24.. WHO: Geneva, 1991.

12 Ngoumou P, Walsh JF. A Manual for Rapid Epidemiological Mapping of Onchocerciasis, TDR/TDE/ONCHO/93.4. WHO: Geneva, 1993.

13 Community Directed Treatment with Ivermectin (CDTI). A practical Guide for Trainers of Community Directed Distributors. APOC: Ouagadougou, 1998.

14 Drameh PS, Richards FO, Cross C, Etya'ale DE, Kassalow JS. Ten years of NGDO action against river blindness. Trends Parasitol 2002; 18: 378-380.

15 Tarimo E. Final Report of Sustainability of Community Directed Treatment of Onchocerciasis with Ivermectin. APOC: Ougadougou, 2000.

16 Amazigo UV, Obono OM, Dadzie KY. Monitoring community directed treatment programmes for sustainability: lessons from the African Programme for Onchocercaisis Control (APOC). Ann Trop Med Parsitol 2002a; 96(Suppl 1): S75-S92.

17 Homeida MMA, GoeppI, Ali M, Hilyer E, Mackenzie CD. Medical acheivements under civil war conditions. Lancet 1999; 354: 601.

18 Hopkins AD. Distribution d'ivermectine dans les pays en conflit. Cahiers Santé 1998; 8(1): 72-74.

19 Mectizan Donation Programme. Mectizan Treatment in Areas where Loaisis and Onchocerciasis are Co-endemic. MDP: Atlanta, 1999.

20 Duke BOL, Zea-Flores G, Castro J, Cupp EW, Munoz B. Efects of three month doses of ivermectin on adult Onchocerca volvulus. Am J Trop Med Hyg 1992; 46: 189-194.

21 Gardon J, Boussinesq M, Kamgno J, Gardon-Wendel N, Demanga N, Duke BOL. Effects of standard and high doses of ivermectin on adult worms of Onchocerca volvulus: a randomized control trial. Lancet 2002; 360: 203-210.

22 Emukah EC, Osuoha E, Miri ES, Onyenama J, Amazigo U, Obijuru $\mathrm{C}$ et al. A longitudinal study of impact of repeated mass ivermectin treatment on clinical manifestations of onchocerciasis in IMO state Nigeria. Am J Trop Med Hyg 2004; 70(5): 556-561.

23 Seketeli A, Adeeoye G, Eyamba A. The challenges and achievements of the African Programme for Onchocerciasis Control (APOC). Annals of Trop Med Parsitol 2002a; 96(Suppl. 1): S15-S28. 